\title{
Hospital responses to COVID-19: evidence from case studies to support future healthcare design research
}

\author{
Nicoletta Setola, Eletta Naldi, Maria Vittoria Arnetoli, Luca Marzi \\ and Roberto Bologna \\ Department of Architecture - TESIS Centre, Università degli Studi di Firenze, \\ Florence, Italy
}

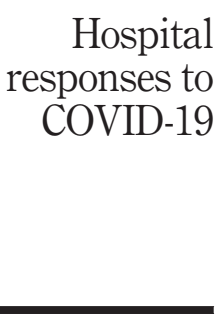

Received 11 March 2021 Revised 2 July 2021 Accepted 5 July 2021

\begin{abstract}
Purpose - The Covid-19 pandemic has placed health-care systems and their facilities throughout the world under immense pressure. The pandemic has highlighted the crucial role of health-care facilities design in looking beyond the ongoing crisis and considering how hospitals can better prepare for unexpected future health situations. This study aims to investigate how hospitals reacted to the crisis in terms of their physical spaces, which architectural features permitted the necessary transformations, and how this data can inform hospital design research in the future.
\end{abstract}

Design/methodology/approach - The research adopted a qualitative and multi-method approach to case studies. Data was collected directly (field survey and interviews) and indirectly (literature, periodicals, specialised websites, webinars, conferences and forums), and a strengths, weaknesses, opportunities, threats analysis supported the data evaluation.

Findings - Hospitals' responses to the crisis were guided by a host of variables depending on the specific intervention context and risk scenario. Some key issues emerged as particularly meaningful to drive future research in hospital design, namely, architectural typology, layout and spatial proximities, technological systems, the quality of care spaces, the role of public spaces, facility management tools to drive the transformation, territorial health care networks and new technologies.

Originality/value - The paper suggests that the current crisis can be transformed into an opportunity, in terms of research and innovation, to rethink and improve the quality and efficiency of health-care spaces, restoring their crucial role of promoting health by design.

Keywords Architecture, Transformation, Layout, Environment, Public spaces, Health

Paper type Research paper

\section{Introduction}

The Covid-19 pandemic has placed some health-care systems throughout the world under immense pressure and stretched others beyond their capacity (WHO, 2020). Health-care systems turned out to be largely unprepared to accommodate a sudden and prolonged peak of, particularly infectious patients. The pandemic highlighted the organisational and structural criticisms of hospitals and underlined their lack of resilience and flexibility in terms of spaces (EXPH-Expert Panel on effective ways of investing in health, 2020): many of them quickly reached their critical limits on the use of space and resources to treat patients with severe symptoms, stem the spread of the virus among patients, visitors and hospital staff and ensure the continuity of ordinary services. The challenge consisted not only of creating and/or finding enough space and resources to treat Covid-positive patients but also

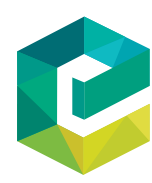


of preventing all other users from being infected while still maintaining the normal daily services.

The pandemic unveiled the crucial role of health-care facilities design in looking beyond the ongoing crisis and considering how health-care systems can better prepare for unexpected future health situations (Bologna et al., 2020). Health-care design, supported by the disaster risk management theory, can address unforeseen situations such as pandemics and catch the so-called "window of opportunity" (Brundiers and Eakin, 2018), ensuring health-care systems are better prepared to address possible future challenges.

Simultaneously, the various latent fragilities of health-care systems, which existed even before the onset of the pandemic (OECD/European Union, 2020), could be tackled by creating improvements that are beneficial even under normal conditions.

In the ongoing pandemic, the design of health-care spaces is one of the most challenging factors, considering both dimensional and distributional aspects, especially in terms of connections, accesses and flows within hospitals, and technological elements, such as the management of equipment and installed systems.

Much of the research on hospital buildings over the years has investigated how the physical environment influences people's health, how it can accelerate the healing process in patients, promote health by encouraging healthy lifestyles and influence the efficiency of health-care workers and, consequently, the effectiveness of care (Verderber, 2010). Therefore, hospital architecture, through optimised layouts based on user needs, high quality spaces and indoor comfort, has an impact on the psycho-physical well-being of patients, operators and carers, in particular reducing their stress and anxiety and increasing their perception of safety (Wagenaar, 2006).

This research led to a series of indications for hospital design based on the best scientific evidence obtained to support designers with guidelines, definitions of specific requirements and technical recommendations to create optimal healthy spaces (Ulrich et al., 2010; NicklWeller and Nickl, 2013; Del Nord, 2006; Setola and Borgianni, 2016). These contributions span different intervention scales, from systems through to buildings, units and individual rooms and different types of sources, from scientific papers to hospital construction manuals and recommendations on specific areas, for example, operating rooms, birth spaces, intensive care and hospital rooms (Wagenaar et al., 2018; Meuser and Labryga, 2019; Joseph et al., 2017; Denham et al., 2018; Rashid, 2015; Foureur et al., 2011; Setola et al., 2018).

Some of these requirements and evidence could be questioned or enriched by the pandemic experience. Even discussions that have long been critical to health-care planning and that now seem consolidated - such as the choice of building type, single versus double rooms, strict isolation or mixed flows (Verderber and Fine, 2000; Setola, 2013) - could now be seen through different eyes.

This paper presents the initial results of an investigation into the effectiveness of the different types of emergency responses adopted in a panel of international case studies, providing the basis for a design contribution to the future management of global health crises. The investigation was conducted by the authors, who have been involved for many years in the fields of health care and emergency design research. In particular, they were involved from April 2020 in the international initiative Beyond Covid-19 Task Force, established by the International Hospital Federation (IHF) to support hospitals in times of crisis (IHF, 2020).

Observing the main transformations to the environment and management adopted by health-care organisations, this study identified three types ( $\mathrm{T}$ ) of response to the crisis according to specific local contexts: 
- T.1 Re-configured health-care facilities. Hospitals that treated Covid patients by making spatial, technological and organisational adaptations such as reorganising accesses, flows and routes to separate Covid-positive patients from others or altering layouts and wards to create dedicated Covid areas;

- T.2 Emergency temporary structures. Temporary buildings created with containers, tents or prefabricated modules were set up near the hospitals, differing in their size and constructive complexity;

- T.3 Re-purposed existing structures. Non-health-related urban structures (e.g. exhibition and cultural centres or fair pavilions), chosen for the availability of empty spaces that could be equipped in a short time, were temporarily adapted by setting up care services.

In addition to these three responses, the reuse of abandoned health-care facilities was identified as another option to manage the pandemic, but rarely applied by health-care organisations due to the prompt response required, the complexities of upgrading work and a general scarcity of information about the spaces and facilities that had been abandoned for a long time.

The actions undertaken were all urgent solutions, which meant there was no time to assess the real resilience of the facilities and the most appropriate alternative solutions for their transformation to fit the specific contexts (Capolongo et al., 2020). The case studies represented a significant source to assess the transformations and their effectiveness according to the context, to extrapolate and discuss key issues related to health-care design research which can inform the adaptability and flexibility of health facilities.

The current crisis can be transformed into an opportunity, in terms of research and innovation, to rethink and improve the quality and efficiency of health-care spaces, restoring their crucial role of promoting health by design, defined in terms of planning, execution and management.

\section{Method}

The design study involved multi-method qualitative data collection and analysis, conducted from March to October 2020. The emergency situation and the severe restrictions of the lockdown imposed a qualitative and dynamic approach to gathering data and its analysis due to the variety of the available information, which was not always complete and continuously changing. Case study data was collected mostly via the web, but the research group expanded the research to one-on-one interviews with some health managers and field observation because of pre-established partnerships.

\subsection{Data collection}

The research group collected information about the spatial and organisation actions adopted by hospitals, focussing on countries with a developed health-care system. Both direct and indirect methods were used to obtain data.

Direct data collection consisted of field surveys in hospitals and informal conversations with the Technical Office of the hospitals willing to speak to the authors. Indirect data collection consisted of gathering information available in literature and periodicals, on the websites of health-care organisations and design studios, and in particular webinars, conferences and forums presenting international case studies on Covid-19 and health-care management and planning, organised by several institutions (i.e. Italian Society of Health care Architecture and Engineering (SIAIS), Politecnico di Milano with the Italian National Centre for Building and Hospital Technology (CNETO) and the College of Engineers and Architects of Milan, Architects for Health and SALUS, IHF and European Health care Design). 
The data acquired by the research group differed in terms of the type and depth of the information. In fact, on the web, it was easier to find generic data about timing, procedure and numbers, while the health-care design webinars provided more detailed and technical insights from health-care professionals, architects and engineers.

\subsection{Selection and categorisation of the case studies}

The case studies were selected on the basis of: their application of the three types of responses (T.1-T.3) (Figure 1); their developed health-care systems and economic context; the availability and accessibility of information during the emergency period.

For each type of response, the research group defined the relevant data dividing it into architectural data concerning spatial and technological transformations, and health-care organisation regarding staff management, logistics and health services (Figure 2). Although the health-care systems vary significantly across the contexts considered, a common evaluation framework was defined focussing on spatial characteristics and elements related to architectural and organisational strategies.

The identified types and different responses were summarised in specific analysis sheets describing the main transformations implemented.

\subsection{Evaluation of the response types}

After the assessment of the case studies supported by the experience of the research group, the three response types were evaluated through a strengths, weaknesses, opportunities, threats (SWOT) analysis and some key issues for the future development of health-care design research were proposed.

\section{Case studies data}

The survey collected 29 case studies, primarily from Italy but also from other European countries (Spain, Germany and UK), the USA, China, Canada and Israel, defining a broad range of interventions.

\subsection{Relevant data of T.1 re-configured health-care facilities}

The architecture-related relevant data of T.1 revealed the extent of the transformations made to the interior and exterior spaces of buildings.

Figure 1.

Overview of the selected studies according to the three types of emergency response

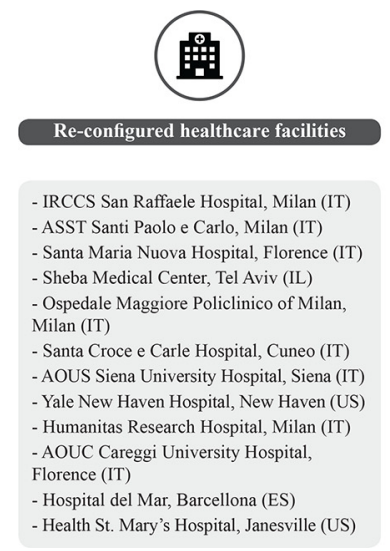

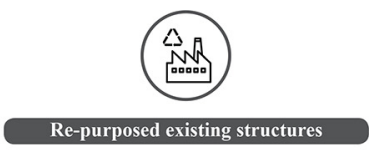

Field Hospital, Cremona (IT)

- Field Hospital, Piacenza (IT) - Samaritan's Purse Field Hospital, NY (US)

- Field Hospital Spallanzani, Rome (IT)

- Covid Center Ospedale del Mare, Naples (IT)

Covid Hospital, Wuhan $(\mathrm{CN})$

- CURA Pods, Turin (IT)

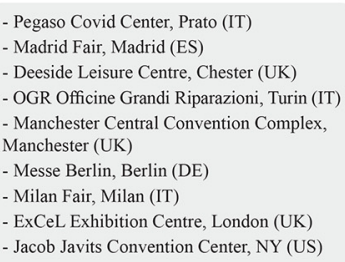




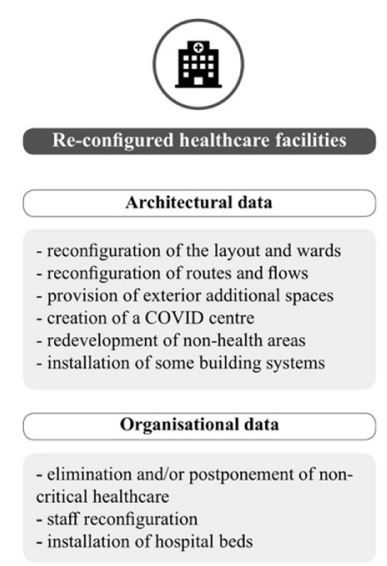

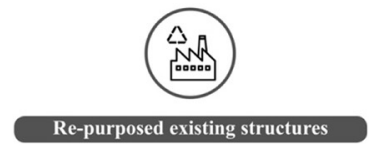

Architectural data

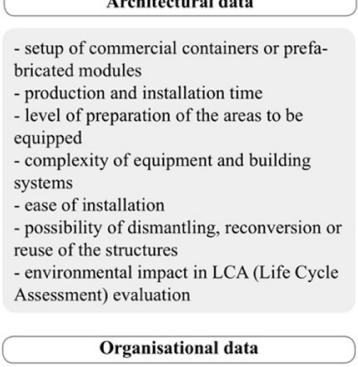

- type of care provided

- management of the relationship with the

referring hospital

- staff required

- number of hospital beds obtained

- bed occupancy rate

Architectural data
- type of structure and designated use
(disused or used structure)
- location with respect to the referring
hospital
- reconversion time
- need for various types of specialised labour
- surfaces involved
- implementation or installation of
equipment and building systems
- incremental developments of the response
- possibility of dismantling, reconversion
or reuse of the structures
$\quad$ Organisational data
- type of care provided
- management of the relationship with the
referring hospital
- staff required
- volunteers and organisations/associations
- number of hospital beds obtained
- bed occupancy rate

\section{Hospital responses to COVID-19}

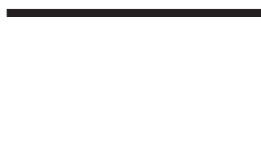

Figure 2.

Relevant data related to each response type divided into architectural and health-care organisational aspects

A key issue was how to reorganise the layouts and units to obtain separate Covid areas when the Department of Infectious Diseases was full. There was a need to find beds for Covid patients, especially in intensive care, which were obtained mainly by transforming operating rooms/surgery units (e.g. in S. Croce e Carle Hospital, Policlinico of Milan) or converting Day Surgeries (e.g. Azienda Socio Sanitaria Territoriale (ASST) Santi Carlo e Paolo), Day Hospitals (e.g. Hospital del Mar) or sport centres (e.g. Istituto di Ricovero e Cura a Carattere Scientifico (IRCSS) San Raffaele Hospital).

Another key point concerning adjustments and changes to "foot traffic" routes and flows to separate positive patients from non-infected staff, patients and materials. Many strategies were adopted, with the most relevant and common concerning the creation of dedicated routes for Covid patients who had separate entrances from outside the building. For example, in Yale New Haven Hospital, the need to reassign the entrances to the pavilions based on infection status (triage, infectious and non-infectious entries) emerged. Other expedients were the decision to limit access to the facility by checking the temperature of everyone entering the buildings (e.g. IRCSS San Raffaele Hospital and Humanitas Research Hospital) or using special demarcations and signage to guide patients with symptoms along special routes to testing areas. For this purpose, many hospitals set up additional exterior spaces near the entrances, i.e. temporary tents for pre-triage for people entering the building, such as Santa Maria Nuova (SMN) which received a tent from the Civil Protection to check symptoms. At AOUC, the entrances to the pavilions had a check-point and covered external routes to protect users in the queues caused by the detection procedures. In AOUS, a prefabricated pre-triage check-in box was set up in the area outside the accident and emergency (A\&E) with dedicated staff controlling access to it.

Moreover, some health-care facilities decided to create Covid centres, buildings entirely dedicated to Covid care which consisted of entirely re-purposed pavilions, as at AOUC, where the surgical clinic pavilion was dedicated to emergency or AOUS where a Covid unit was set up in the newly built block near the intencive care unit (ICU) of the A\&E. 
Alternatively, underused or abandoned units were converted for Covid care, for instance, at SMN, Hospital del Mar or ASST Santi Carlo e Paolo where an abandoned surgery unit was transformed into an ICU. Elsewhere, some facilities redeveloped non-health-care areas within their hospital campus, such as gyms (IRCCS San Raffaele Hospital and Hospital del Mar) or underground parking floors, for instance, the one at Sheba Medical Centre, originally designed to serve as an emergency hospital for wartime, in three days was transformed into a Covid ICU to implement the new model of care using inpatient telemedicine.

Finally, many facilities, especially the older ones, had to implement some technological systems. The need for medical gases (especially oxygen supplies) imposed some changes or the installation of distribution plants, along with alterations to the air treatment system to depressurise air and obtain negative pressure rooms. This was the case at Carle Hospital, where the pipes and storage capacity of the oxygen tanks were doubled or S. Paolo Hospital where a second oxygen tank, a double reduction framework and a new medical air system were introduced.

According to the health-care organisation data, the most characteristic element of the response inside the health-care facilities was the elimination or deferment of health services due to the reconversion of some units and staff reorganisation. These interrupted services, with "deferrable schedules", were low-priority diagnostics and surgery and outpatient activities. In fact, the search for health-care professionals for Covid care mostly involved already working professionals who were transferred to Covid units or new hires (e.g. Policlinico of Milan) also among young doctors undergoing specialised medical training or retired doctors. Finally, the buildings had to obtain equipment, such as personal protective equipment (PPE), ventilators or beds in ICU and recovery wards.

\subsection{Relevant data of T.2 emergency temporary structures}

The use of temporary prefabricated structures, such as modules, containers or tents, highlighted crucial design and process aspects, especially relating to the environmental and socio-economic sustainability of the entire life cycle.

The architectural data covers various aspects, firstly, the possibility to set up the widely available commercial containers (e.g. Covid Hospital in Wuhan) or design specific prefabricated module services and systems for clinical aspects of coronavirus disease (e.g. CURA Pods in Turin). Unlike tents, the production and installation time required for containers and modules had to be considered, which took up to two weeks to one month, implying specialised labour depending on the health care plan and the preparation of the equipped area, including the provision of an access road and connection services. The second aspect, in particular, could result in a delayed response and rising costs. The number of preliminary operations depended both on the features of the available installation area (the Spallanzani parking lot differs from the extraordinary "invasion" of Central Park in New York) and the level of care provided (ICU in CURA Pods compared to the London Health Science Centre in Canada) which determined the complexity of the equipment and technological systems. The suitability of the different structures for multiple levels of care was relevant, for example, tents favoured time-limited processes while temporary modules provided more complex health-care treatments. Finally, the preventive definition of post-emergency scenarios is fundamental to consider the environmental sustainability of the intervention, focussing on alternative methods of disposal, reuse or reconversion.

As regard the organisational aspects, the key issues are the type of care provided and the severity of the patients to be treated who require either intensive care (e.g. Covid Centre in Naples) or just recovery services (e.g. Field Hospital in Cremona), and whether or not there 
are other specialised care departments. The level of care is strictly linked to the management of the relationship with the referring hospital in terms of health-care services, furniture, equipment (Spallanzani Field Hospital is located at the Hospital entrance while Piacenza's field hospital is located in a military area).

The staff required for these additional facilities was another important point. In fact, in most cases the professionals were not sufficient, so they began to rapidly hire employees. This data is determined by the number of hospital beds used, and whether there was an ICU or sub-ICU (e.g. in Naples there were not enough staff members to manage the 72 ICU beds). To conclude, another important issue is the bed occupancy rate, that is the number of Covid patients treated in the new emergency temporary facility.

\subsection{Relevant data of T.3 re-purposed existing structures}

The third response type demonstrates the potential of the temporary conversion of large available spaces pertaining to buildings used for sports, cultural activities or fairs. The vast dimensions of the interior spaces, usually empty because of their use as a temporary exhibition or event spaces, meant they could easily be equipped with partitions and beds, and even provided with complex equipment. The first piece of relevant architectural data concerns the building type and its ordinary use. For the most part, congress centres were used (e.g. Messe Berlin, ExCeL Centre and Madrid Fair) but also train stations (Manchester Central) or industrial structures [officine grandi riparazioni (OGR) in Turin] were repurposed into cultural spaces, and even a research centre under redevelopment (Pegaso Covid Center in Prato). As regard T.2, the location was a key factor for supplies and staff, considering not only the distance from the referring hospital (Manchester Central is beside the National Health Service Nightingale Hospital North West, while Milan Fair is $14 \mathrm{~km}$ from the Policlinico of Milan) but also the urban context (OGR is in the compact city centre of Turin whereas the Deeside Leisure Centre is in a suburban area). Moreover, important aspects were the reconversion time (at Deeside Centre it took under three weeks to set up 430 beds, while in Berlin it took six weeks to set up 500 beds), the surface area involved, which in many cases was oversized (for instance, $87,500 \mathrm{~m}^{2}$ in the ExCeL Centre or 25,000 $\mathrm{m}^{2}$ in Milan Fair), and the need to implement or install equipment depending on the health service (as in Javits Centre). Finally, it is important to consider the incremental developments of the response which frequently followed the worsening of the health crisis (for instance, Milan had three planned phases), not underestimating the need to dismantle or reuse the equipment.

The relevant health-care organisation data - the type of care provided, the relationship with the referring hospital, the staff necessary to run the facility, the number of beds set up, the bed occupancy rate and if volunteers or associations were involved, for example, in Madrid firefighters, the Army and Fair staff were involved arriving at 300 people between professionals and volunteers - mostly coincides with T.2, as both T.2 and T.3 response strategies were based on managing Covid patients outside the main hospital facilities.

In addition, general considerations could be made regarding the economic impacts of the transformations and the post-pandemic evolutions. Certainly, the intervention costs of the second and third Ts were more consistent than the first and, from the viewpoint of a circular economy and waste reduction, the capacity to reuse or reconvert the temporary structures and equipment determines their impact on the long-term post-emergency period.

\section{Results}

The strengths, weaknesses, opportunities and threats in the SWOT analysis distinguish between architectural and health-care organisation aspects (Tables 1-3). 
T.1 Re-configured health-care facilities

Strengths

- Architectural:

- Containment of spatial and structural interventions consisting mainly of spatial reversible adjustments to isolate and contain Covid;

- Quality and humanisation of spaces;

- Cost saving in space adjustment;

- No need for specialised equipment and

labour for internal partitions

- Health-care organisation:

- Decision-making and management

autonomy because of the possibility of tackling the emergency with internal strengths;

- Ease and flexibility in staff management as most already work at the facility;

- Reduced operating costs compared to the

creation of new Covid spaces

Opportunities

- Architectural:

- Dynamism and responsiveness compared to

the creation of new Covid spaces;

- Some specific surgery unit shapes to facilitate the shift to Covid treatment (mainly ICU), for example, a concentric layout of the hallways surrounding the operating rooms meant the external one could be used as a Covid route

- Health-care organisation:

Table 1.

SWOT analysis of T.1 re-configured health-care facilities
- Easy response to possible patient needs through the presence of various departments and types of services able to provide comprehensive health assistance (not only related to Covid)
- Architectural:

Weakness

- Low degree of transformability hindered by the building conformation;

- Technological system adaptation of air and medical gas plants (mainly oxygen)

- Health-care organisation:

- Structural reconfiguration at the functional level because of layout alterations and staff organisation;

- Conversion of some operating units for Covid treatment;

- Interruption or decrease of deferrable health services to dedicate space and professionals to the Covid emergency

- Architectural:

Threats

- Degree of transformability depends on the urban surroundings;

- Proximity between Covid units and others (e.g. when infected areas depend on diagnostics placed outside);

- Diversifying routes and flows depending on the redundancy and conformation of horizontal and vertical routes;

- Ensuring staff spaces (vestibules, anterooms for donning and doffing, rest rooms and storage)

- Health-care organisation:

- Possible presence of mixed routes and the consequent need for sanitisation and ad hoc protocols

The aim of the SWOT analysis was not to define the three responses as alternative options for health-care facilities, but to identify from each type the practices to implement, and to combine them in key issues representing the direction of future research to support adaptations in health-care facilities.

\section{Discussion}

The SWOT analysis highlighted the complexity of the problem: the hospitals' response types were guided by a number of variables. Some of them depended on the specific context of the intervention, such as the health-care system model, the territorial health-care network, the physical characteristics of the facility (age, size, architectural type, etc.) and the care services provided. Others depended on the risk scenario, for example, the type and intensity of the risk determining the importance of incremental and modular interventions related to increasing degrees of risk proportional to the number and severity of the patients to be managed.

In spite of the complex framework of the current and evolving situation, we find ourselves in, the paper aims to highlight and discuss some key issues deriving from the analyses described 


\section{T.2 Emergency temporary structures}

Strengths

- Architectural:

Mobile and relocatable units;

Rapid installation;

Easily incrementable and expandable;

Diversified spatial and functional configurations

- Health-care organisation:

Additional spaces enable the referring hospital

to keep its services intact;

Easier containment of infection due to the absence of users moving between Covid and Covid-free areas

Opportunities

- Architectural:

Less impact on the referring hospital regarding accesses, flows and units;

Structure designed for specific health needs; Maintaining the temporary structure post emergency

- Health-care organisation:

Integrating the temporary structure with the health-care systems to respond to pre-existing needs
- Architectural:

Weakness

High intervention costs;

Guaranteeing the integration of technological systems and equipment especially for ICU;

Installation of all necessary technological systems in the area;

Need for specialised equipment and labour to manage the building site;

Poor quality and low level of humanisation of spaces

Health-care organisation:

Increased operating costs;

Additional staff required, from health-care professionals to cleaning staff

Threats

- Architectural:

Location of the area to be equipped in relation to the functional areas of the referring hospital;

Disposal of the structure after the emergency;

High environmental impact of the entire life cycle

- Health-care organisation:

Difficulty of managing additional health issues not catered for by the structure

Hospital

responses to

COVID-19 
above, to inform health-care design research in the near future with a view to supporting the resilience of health organisations and facilities facing pandemic events.

\subsection{Architectural typology}

The type of building (tower monoblock, flat monoblock and pavilions) plays a fundamental role in allowing flexible transforms. Consideration should be given to this and the long-standing discussion of the best type to be adopted for the hospital of the future (Cox and Groves, 1990; Verderber and Fine, 2000; Capolongo et al., 2019). Pavilions, originally created to combat the spread of infection, allow patients to be isolated more easily, dedicating a single block to Covid functions. On the other hand, this type makes it more difficult to control and limit access to the hospital area than a monoblock. In the flat monoblock, the biggest issue is how to achieve isolation, by creating independent units or compartmentalisation wings and separating flows. In the tower block, compartmentalisation can occur by separating the floors, but problems may arise with the separation of the vertical connections, which may not be sufficient.

\subsection{Layout and spatial proximities}

It is also necessary to reflect on the organisation of the layout and proximity relationships between functional areas in hospitals (Fermand, 1999). A flexible layout should increase hospital capacity through expandable departments, converting existing units into Covid units, creating separate access points, setting up beds in patient rooms and providing additional spaces for vestibules, all while allowing the continuity of ordinary care.

Some types of spatial layouts worked better than others. For example, in some emergency unit layouts, it was possible to separate flows and create spaces where people could wait for test results. These emergency units also worked well because they had a specific area for diagnostic services.

While operating theatres with a concentric layout with clean and dirty paths made it easier to separate infected flows and at the same time use a part of them for normal services and a part for surgeries for Covid patients. This cannot happen in operating room models that have mixed dirty and clean flows.

Some key barriers to transformation were also found in spatial layouts that lacked certain spaces in the hospital, for example, a lack of spaces for staff working in Covid areas to properly don and doff PPE, rooms too small to store technical equipment to support intensive care, a shortage of storage and waste areas for supplies and the need to provide rest rooms for staff.

Some hospital areas, such as Maternity and Oncology, need to be accessible and clean even during pandemic-related transformations. At times, this led to the duplication of entire functional areas, such as Covid and normal Maternity or it occurred to the detriment of physiological pathways.

In rethinking the proximity relationships and new layout models for specific functional areas, the flows and channels of circulation of the entire hospital should also be rethought, paying attention to the dimensions of the corridors, which could become storage for medical equipment or spaces for double circulation.

\subsection{Technological systems}

A big issue was the adaptation of plants (for example, depressurising air, inversion of air, etc.) and the presence of medical gas because of the higher consumption of oxygen for intensive care. Consideration should be given to the possibility of installing technological systems (airflows that can change room air pressure and medical gas plants) in convertible units but also to set up connection points for plant systems in external areas such as parking lots, sports centres or conference halls as potential areas for care expansion. 
All the spatial transformations should ensure, even in demanding circumstances, a quality environment and adequate levels of equipment according to the humanisation of care spaces principles (Bosia and Darvo, 2015). In situations like that of the pandemic where patients are not allowed visitors and staff members are working under stressful conditions, the need to be surrounded by beautiful and regenerative places becomes increasingly evident. It takes a great deal for designers to come up with humanised solutions capable of providing the necessary flexibility. Perhaps, the research could help to define new requirements and standards in light of the pandemic experience. The hospital room is certainly a space to work on in the future: planning requirements for the "universal patient room" with a spatial conformation supporting the spectrum of acuity and surge capacity (considering the shape, size, technological systems, complementary space and additional equipment); patient rooms should be able to accommodate one or two additional beds and larger rooms with added gases should be able to accommodate double occupancy for surges; the quality of the spaces should be assessed in terms of comfort and usability to support an effective health-care response and the quality perceived by patients and operators.

\subsection{Role of public spaces}

Much has been said about the role of public spaces in hospitals in previous research (Setola and Borgianni, 2016), but, perhaps, new functions and requirements need to be added. As public spaces represent the access points to hospitals and are usually large in size, they become an important resource in emergency situations. In fact, buffer zones in hospitals, ancillary areas that do not host medical assistance activities in ordinary circumstances, could be converted during emergency situations if they are designed with suitable dimensional and technical requirements for health-care services. The types of spaces that can be converted for emergency use are internal and external public spaces, such as entrance plazas, parking lots, green areas, hospital streets, halls and gyms.

The feasibility of the strategy for external areas depends again on the specific type of building (e.g. monoblock or pavilion hospital) and its setting (e.g. hospital located in a campus with many empty areas or in a densely built urban context). From this perspective, the design of temporary additional elements (tents, containers or prefabricated modules) can result in the incremental provision of services as an extension of the building. These are minimal units that can be used as temporary additional spaces of the hospital or reversible buildings for entering, waiting and sorting, first aid and triage, to be placed in the outdoor spaces adjacent to the hospital (entrance square, porches, parking lots, etc.). Considering the accessibility and mobility connections of the buffer zones, outdoor spaces next to the hospital such as porches, squares and parking lots should be capable of accommodating light structures to facilitate entry, screening and waiting.

While as regard the internal public spaces, some considerations should be made on the strategic positioning, configuration and dimensioning of spaces such as entrance halls, conference rooms and loading docks for their potential conversion and the possibility to accommodate controlled access points and differentiated paths into the hospital. In fact, in some cases, the control of the entrance was difficult. In most hospitals, a unique checkpoint gate was set up to measure people's temperature with a thermo scanner and divide the flows.

Reconsidering the dimensions of waiting areas and their position is another key point to ensure physical distances and safe queuing in the case of infections; new furniture could be designed for this purpose. 
Thus, new requirements for the internal public spaces would be useful to plan accesses, flows, vertical and horizontal connections, separate infectious and non-infectious entrances and screening facilities.

\subsection{Facility management tools to drive the transformation}

The analysis showed how important it is to manage the spatial, structural and systems data available to quickly plan the interventions to be carried out. For this reason, it would be necessary to invest in the research and development of assessment and decision-making tools to support the daily management of hospital settings and allow the evaluation of optimal alternative hypotheses in the case of transformation.

At the international level, several tools have been proposed to improve quality, reduce risks and safety, and increase efficiency, accountability or sustainability during the pandemic. These tools specifically developed for Covid-19 (Baldwin, 2020; Agarwal et al., 2020; De Georgeo et al., 2021; Yen et al., 2020) provide more or less accurate information on certain ideal scenarios but more investigation is needed to define their scalable features in specific contexts and according to available resources.

\subsection{Territorial health-care networks and new technologies}

The above-mentioned considerations must be viewed in terms of two fundamental trends, the relationship between facilities and territorial health-care networks and the integration of new technologies into care spaces and services.

Considerations on the need to implement the resilience of each building and health-care systems cannot be separated from the reorganisation of the welfare system at the territorial level, as this is a prerequisite for the planning stages (EXPH-Expert Panel on effective ways of investing in health, 2020). In fact, given that the general territorial system permits the differentiation of buildings types according to services by assigning them a level of acuity, in emergency conditions this means different functional responses and spatial organisation, triggering virtuous mechanisms of architectural and social redevelopment. The spread of Covid-19 has demonstrated that territorial reorganisation should ensure greater capillarity and accessibility of services following a decentralisation and decongesting process; the stratification of services by the intensity of care and integration between health services and primary care produced a more effective response.

The other macro trend is the enhanced digitalisation of the management of the physical facilities and services. In fact, Covid-19 helped to speed up the transition towards the integration of new technologies and Artificial Intelligence in health-care services by changing how health-care spaces, staff and patients interact (Gunasekeran et al., 2021).

The integration of new functions, the creation of new spaces and the definition of their dimensional and environmental requirements provide useful inputs for new design paradigms implementing technological innovations such as telemedicine (video consultation and videoconferences), applications for smartphones, wearable technology, smart sensors, the use of robots, camera analytics, real time location services and automation processes. The physical space of single buildings and the territorial planning of the whole network must be rethought according to these changes, adapting to the needs of digital health-care and the dematerialisation of operator-patient interactions and data management.

\section{Conclusions}

How hospitals reacted depended on the intensity of the pandemic and how the territorial assistance network worked, but the possibility to functionally reorganise the units, flows and accesses also played a key role in responding with dynamism and autonomy. This 
meant implementing the choice of convertible units inside the hospitals, new layout solutions concerning spaces and the adaptation and management of technological systems.

In particular, some architectural and technical characteristics of hospitals, discussed above, triggered the process of transforming the existing spaces. When those features were absent, the changes were more difficult, and this led to the search for spaces outside the hospital to complete the requirements.

Integrating the paradigms of strategic planning into health-care design to manage emergency situations would increase the resilience of hospital services to disruptive events, such as pandemics, addressing the vulnerability factors.

A preventive vision, based on the definition of multiple and alternative intervention scenarios, could combine the ordinary needs of the design of physical buildings and immaterial services with the extraordinary ones, now more and more frequent, strengthening the adaptability reserves of buildings, and therefore their ability to respond to shock and stress without suffering damage or interruptions but, on the contrary, ending up with better systems than they had prior to the event.

Looking at the results of how hospitals responded to the Covid-19 pandemic, it is clear that increasing the flexibility and adaptability of the individual hospitals and of the overall network of health-care facilities and services is a priority.

To achieve this objective, health-care design research can make a strong contribution to developing building interventions that guarantee fast and efficient delivery of health care, and at the same time adequate quality in terms of the comfort and usability of the spaces even under extraordinary conditions.

Although many questions remain open, also depending on the evolution of the ongoing pandemic, this paper suggests key issues to address future research on healthcare architecture. The adaptation of health-care facilities to Covid-19, as well as other kinds of risks, can further facilitate the transition towards the target of the Green Deal and Artificial Intelligence.

\section{References}

Agarwal, A., Nagi, N., Chatterjee, P., Sarkar, S., Mourya, D., Sahay, R.R. and Bhatia, R. (2020), "Guidance for building a dedicated health facility to contain the spread of the 2019 novel coronavirus outbreak", Indian Journal of Medical Research, pp. 177-183.

Baldwin, E. (2020), "AIA's COVID-19 task force creates design guide to retrofit buildings for alternative care", available at: www.archdaily.com/937331/aias-covid-19-task-force-creates-design-guide-toretrofit-buildings-for-alternative-care (accessed 15 January 2021).

Bologna, R., Setola, N., Marzi, L., Naldi, E. and Arnetoli, M.V. (2020), "COVID-19: sparking a new revolution in hospital design?", available at: www.ihf-fih.org/2020/12/07/covid-19-sparking-anew-revolution-in-hospital-design/ (accessed 15 January 2021).

Bosia, D. and Darvo, G. (2015), "The guidelines for the humanisation of care facilities", TECHNE - Journal of Technology for Architecture and Environment, No. 9, pp. 140-146, doi: 10.13128/Techne-16115.

Brundiers, K. and Eakin, H.C. (2018), "Leveraging post-disaster windows of opportunities for change towards sustainability: a framework", Sustainability, Vol. 10 No. 5, pp. 1-18.

Capolongo, S., Cocina, G., Gola, M., Peretti, G. and Pollo, R. (2019), "Horizontality and verticality in architectures for health", TECHNE - Journal of Technology for Architecture and Environment, No. 17, pp. 152-160, doi: 10.13128/Techne-24028.

Capolongo, S., Gola, M., Brambilla, A., Morganti, A., Mosca, E.I. and Barach, P. (2020), "COVID-19 and healthcare facilities: a decalogue of design strategies for resilient hospitals", Acta Biomedica, Vol. 9, pp. 50-60. 
Cox, A. and Groves, P. (1990), Hospitals and Health-Care Facilities a Design and Development Guide, Rev, 2nd ed., Butterworth Architecture, London; Boston.

De Georgeo, M.R., De Georgeo, J.M., Egan, T.M., Klee, K.B., Schwemm, M.S., Bye-Kollbaum, H. and Kinser, A.J. (2021), "Containing SARS-CoV-2 in hospitals facing finite PPE, limited testing, and physical space variability: navigating resource constrained enhanced traffic control bundling", Journal of Microbiology, Immunology and Infection, Vol. 54 No. 1, pp. 4-11.

Del Nord, R. (2006), Environmental Stress Prevention in Children's Hospital Design, Motta Editore, Milano.

Denham, M.E., Bushehri, Y. and Lim, L. (2018), "Through the eyes of the user: evaluating neonatal intensive care unit design", Herd: Health Environments Research and Design Journal, Vol. 11 No. 3, pp. 49-65.

EXPH-Expert Panel on effective ways of investing in health (2020), "The organisation of resilient health and social care following the COVID-19 pandemic", 25 November 2020 (C) European Union.

Fermand, C. (1999), Les Hôpitaux et Les Cliniques, Architectures de la Santé, Le Moniteur.

Foureur, M., Leap, N., Davis, D.L., Forbes, I.F. and Homer, C. (2011), "Testing the birth unit design spatial evaluation tool (BUDSET) in Australia: a pilot study", Herd: Health Environments Research and Design Journal, Vol. 4 No. 2, pp. 36-60.

Gunasekeran, D.V., Tseng, R., Tham, Y.C. and Wong, T.Y. (2021), "Applications of digital health for public health responses to COVID-19: a systematic scoping review of artificial intelligence, telehealth and related technologies", NPJ Digital Medicine, Vol. 4 No. 1, p. 40, doi: 10.1038/s41746021-00412-9.

International Hospital Federation (2020), "Building the 'new normal': harnessing transformative practices from the COVID-19 pandemic", available at: www.ihf-fih.org/beyond-covid-19-taskforce-reports/ (accessed 15 January 2021).

Joseph, A., Sara Bayramzadeh, S., Zamani, Z. and Rostenberg, B. (2017), "Safety, performance, and satisfaction outcomes in the operating room: a literature review", Herd: Health Environments Research and Design Journal, Vol. 11 No. 2, pp. 137-150.

Meuser, P. and Labryga, F. (2019), Hospitals and Medical Facilities: Construction and Design Manual, DOM Publisher.

Nickl-Weller, C. and Nickl, H. (2013), Healing Architecture, Braun Publishing AG, Schweiz.

OECD/European Union (2020), "Health at a glance: Europe 2020: state of health in the EU cycle", OECD Publishing, Paris, available at: https:/ec.europa.eu/health/sites/health/files/state/docs/ 2020_healthatglance_rep_en.pdf (accessed 13 January 2020).

Rashid, M. (2015), "Research on nursing unit layouts: an integrative review”, Facilities, Vol. 33 Nos 9/10, pp. 631-695.

Setola, N. (2013), Percorsi, Flussi e Persone Nella Progettazione Ospedaliera. L'analisi Configurazionale, Teoria e Applicazione, Firenze University Press, Firenze.

Setola, N. and Borgianni, S. (2016), Designing Public Spaces in Hospitals, Routledge, New York, NY.

Setola, N., Iannuzzi, L., Cocina, G.G., Naldi, E., Escuriet Peiró, R., Morano, S., Branchini, L., Santini, M. and Downe, S. (2018), "Optimal settings for childbirth", Minerva Ginecologica, Vol. 70 No. 6, pp. 687-699.

Ulrich, R.S., Ulrich, R.S., Berry, L.L., Quan, X. and Parish, J.T. (2010), “A conceptual framework for the domain of evidence-based design", Herd: Health Environments Research and Design Journal, Vol. 4 No. 1, pp. 95-114.

Verderber, S. (2010), Innovation in Hospital Architecture, Routledge, New York, NY.

Verderber, S. and Fine, D.J. (2000), Healthcare Architecture in an Era of Radical Transformation, Yale University Press, New Haven and London.

Wagenaar, C. (Ed.) (2006), The Architecture of Hospitals, NAi Uitgevers, Rotterdam. 
Wagenaar, C., Mens, N., Manja, G., Niemeijer, C. and Guthknecht, T. (2018), Hospitals: A Design Manual, Birkhauser, Basel.

World Health Organization (2020), "Attacks on health care in the context of COVID-19", available at: www.who.int/news-room/feature-stories/detail/attacks-on-health-care-in-the-context-of-covid-19 (accessed 13 January 2020).

Yen, M.Y., Schwartz, J., Chen, S.Y., King, C.C., Yang, C.Y. and Hsueh, P.R. (2020), “Interrupting COVID-19 transmission by implementing enhanced traffic control bundling: implications for global prevention and control efforts", Journal of Microbiology, Immunology and Infection, Vol. 53 No. 3, pp. 377-380.

\section{Corresponding author}

Nicoletta Setola can be contacted at: nicoletta.setola@unifi.it

For instructions on how to order reprints of this article, please visit our website: 